\title{
Unknown Radial Distortion Centers in Multiple View Geometry Problems
}

\author{
José Henrique Brito ${ }^{1,2}$, Roland Angst ${ }^{3}$, Kevin Köser ${ }^{3}$, Christopher Zach ${ }^{4}$, \\ Pedro Branco ${ }^{2}$, Manuel João Ferreira ${ }^{2}$, Marc Pollefeys ${ }^{3}$ \\ ${ }^{1}$ Intituto Politécnico do Cávado e do Ave, Barcelos, Portugal \\ ${ }^{2}$ Universidade do Minho, Guimarães, Portugal \\ ${ }^{3}$ Computer Vision and Geometry Group, ETH Zürich, Switzerland \\ ${ }^{4}$ Microsoft Research, Cambridge, UK
}

\begin{abstract}
The radial undistortion model proposed by Fitzgibbon and the radial fundamental matrix were early steps to extend classical epipolar geometry to distorted cameras. Later minimal solvers have been proposed to find relative pose and radial distortion, given point correspondences between images. However, a big drawback of all these approaches is that they require the distortion center to be exactly known. In this paper we show how the distortion center can be absorbed into a new radial fundamental matrix. This new formulation is much more practical in reality as it allows also digital zoom, cropped images and camera-lens systems where the distortion center does not exactly coincide with the image center. In particular we start from the setting where only one of the two images contains radial distortion, analyze the structure of the particular radial fundamental matrix and show that the technique also generalizes to other linear multi-view relationships like trifocal tensor and homography. For the new radial fundamental matrix we propose different estimation algorithms from 9,10 and 11 points. We show how to extract the epipoles and prove the practical applicability on several epipolar geometry image pairs with strong distortion that - to the best of our knowledge - no other existing algorithm can handle properly.
\end{abstract}

\section{Introduction}

When trying to relate images, the robust estimation of the fundamental matrix based on local feature correspondences is a very powerful approach. Stochastic estimation algorithms such as RANSAC can find the correct two-view relation with high probability and at the same time distinguish inliers and outliers to the model (i.e. mismatches). However, this approach relies on the appropriateness of the model, i.e. it assumes that the images strictly obey to the pinhole camera model. In practice however, images can contain significant distortion induced by the lens (system) of a real camera. Consequently, in the literature several camera models and techniques have been proposed to model such distortion [3, $6,16,1,17,4,9]$. However, for automatic registration of images obtained from internet sources or archives, an offline camera calibration phase is not feasible. 
In such cases lens distortion has to be considered directly in the multi-view geometry estimation stage. This was the idea of the undistortion model proposed by Fitzgibbon [6] that has been extended to the radial fundamental matrix by Barreto and Daniilidis [1]. The assumption is that undistortion can be modeled in a radial fashion with respect to a distortion center. The main drawback in both formulations is that the distortion center must be known in advance, which we argue is not practical when images stem from sources like archives or internet photo collections. Using a wrong distortion center renders the whole concept of radial distortion meaningless, although assuming the distortion center to be at the center of the image can sometimes still be a valid approximation. However, in the case of cropped images or images taken with digital zoom no heuristics exist where to place the distortion center. Consequently, in this contribution we generalize the radial fundamental matrix (and all other multilinear multiple view relations) to unknown distortion centers. This is very analogue to the ideal pinhole case where the essential matrix was generalized to the fundamental matrix [5] that could then account for any principal point. Also in the case of the radial fundamental the dimensions of the matrix do not change once the distortion center is considered and linear algorithms require the same number of points for estimating it.

For clarity of presentation we start from the setting where only one of the two images contains radial distortion and analyze the structure of the particular radial fundamental matrix. It will turn out that a change of distortion center acts linearly on the lifted point representation, allowing to do the same generalization for other multi-view geometry relations like homograpy, trifocal tensor and so forth. We then continue to derive different estimation algorithms for our radial fundamental matrix from 9,10 and 11 points that exploit the specific algebraic structure and show how to extract the epipoles. Finally, we prove the practical applicability of the new theory on several epipolar geometry image pairs with strong distortion that - to the best of our knowledge - no other existing algorithm can handle properly.

\section{Previous Work}

For ideal pinhole cameras the essential matrix has been introduced by LonguetHiggins [14] and it allowed efficient computation of the relative pose between two views. However, pre-calibration of these views was mandatory and prevented using this technique for images with unknown calibration parameters since one had to know e.g. focal length and principal point of both cameras. Much later, the introduction of the fundamental matrix $[5,15]$ removed this restriction and allowed to work with unknown images, zoom cameras and led to a whole theory of auto-calibration from images and projective reconstruction (cf. to [10]). Practically, already the original 8-point algorithm from [14] could have been applied to the uncalibrated setting, but due to notation and for historic reasons this was not clear before the proposal of the fundamental matrix. Nowadays, the core of 
Longuet-Higgins algorithm is known as the 8- point algorithm for fundamental matrix estimation [10].

The fundamental matrix applies to ideal pinhole cameras, but real cameras have lenses that sometimes result in distortion of the image and, due to the shape of the lens, this distortion is typically radially-symmetric with respect to a distortion center. Many formulations exist to cope with this problem (e.g. [6, $16,1,17,4,9]$. According to one of the classical distortion models [3] the deformation of an undistorted point into a distorted point (as caused by the lens) is represented by a polynomial equation, but due to the nature of the distortion function it was not easily possible to estimate the inverse of the distortion directly from point correspondences. Fitzgibbon[6] has suggested to directly model the undistortion of a point rather than the distortion and argued that earlier distortion models were as good or bad empiric approximations to the true lens behaviour as an undistortion model might be. Having an undistortion model has the advantage that one can directly work with distorted coordinates, which is what is measured in an image.

However, similarly as in the derivation of the essential matrix of LonguetHiggins, now Fitzgibbon assumed that the distortion center is known beforehand. Later, his model was reformulated into the radial fundamental matrix by Barreto and Daniilidis[1]. They proposed a linear 15 point method to estimate the matrix and recently it has been shown by Kukelova et al. [12] that this view relation can be estimated actually from only 9 correspondences in a minimal solver. All of the above mentioned papers kept the strong requirement that the distortion center needs to be known in advance, which practically prevented the use of these techniques for unknown, cropped images or in the case of (digital) zoom. Li et al. [13] addressed the unknown distortion center problem, but they need a calibration grid or a very high number of noise-free point correspondences, among other restrictions.

In this paper we will show that the position of the distortion center can be absorbed into the radial fundamental matrix in very much the same way as the principal point is absorbed into the fundamental matrix.

\section{The Lifting-Trick for Radial Distortion}

\subsection{Second-Order Radial Distortion Models}

The traditionally used second-order distortion model in computer vision with unknown center of distortion $\left(d_{x}, d_{y}\right)^{T} \in \mathbb{R}^{2}$ describes the radial distortion as

$$
\left(\begin{array}{l}
x_{d} \\
y_{d}
\end{array}\right)=\left(\begin{array}{l}
x_{u} \\
y_{u}
\end{array}\right)+\lambda \tilde{r}^{2}\left(\left(\begin{array}{l}
x_{u} \\
y_{u}
\end{array}\right)-\left(\begin{array}{l}
d_{x} \\
d_{y}
\end{array}\right)\right)
$$

where $\left(x_{d}, y_{d}\right)^{T} \in \mathbb{R}^{2}$ and $\left(x_{u}, y_{u}\right)^{T} \in \mathbb{R}^{2}$ are the distorted and the undistorted point, respectively, whereas $\lambda \in \mathbb{R}$ is the distortion coefficient and $\tilde{r}^{2}=$ $\left\|\left(x_{u}, y_{u}\right)^{T}-\left(d_{x}, d_{y}\right)^{T}\right\|^{2}$ is the squared Euclidean distance between the center 
of distortion and the undistorted point. Eq. 1 is a distortion model since it actually describes the distorted point in explicit form: given the undistorted point $\left(x_{u}, y_{u}\right)^{T}$ and the distortion parameters $\lambda$ and $\left(d_{x}, d_{y}\right)^{T}$, the distorted point can be computed easily by evaluating the right-hand side of Eq. 1 .

Fitzgibbon [6] has proposed a slightly different model, which he showed to be equivalently powerful as the model above, i.e. it provides the same approximation accuracy to the underlying true distortion. However, his model enjoys an interesting property. Specifically, this radial distortion model can conveniently be expressed with homogeneous coordinates

$$
p_{u}=\left(\begin{array}{c}
x_{u} \\
y_{u} \\
1
\end{array}\right) \cong\left(\begin{array}{c}
x_{d} \\
y_{d} \\
1+\lambda r^{2}
\end{array}\right),
$$

with $r^{2}=x_{d}^{2}+y_{d}^{2}$ and where $\cong$ denotes equality up to a scalar multiple. In this paper, we extend his formulation to the case where not only the distortion coefficient $\lambda$ is unknown, but the center of radial distortion $\left(d_{x}, d_{y}\right)^{T}$ as well. In this case, his model can be extended by starting with

$$
\left(\begin{array}{l}
x_{d} \\
y_{d}
\end{array}\right)=\left(\begin{array}{l}
x_{u} \\
y_{u}
\end{array}\right)+\lambda r^{2}\left(\left(\begin{array}{l}
x_{u} \\
y_{u}
\end{array}\right)-\left(\begin{array}{l}
d_{x} \\
d_{y}
\end{array}\right)\right)
$$

where $r^{2}=\left\|\left(x_{d}, y_{d}\right)^{T}-\left(d_{x}, d_{y}\right)^{T}\right\|^{2}$. The only distinction to the model in Eq. 1 is that the distance is now measured between the distorted point and the center of radial distortion. In contrast to the distortion model in Eq. 1 however, Fitzgibbon's model actually is an undistortion model: the right-hand side of Eq. 3 is linear in the undistorted point $\left(x_{u}, y_{u}\right)^{T}$ and hence one can compute an explicit form for this undistorted point given the distorted point $\left(x_{d}, y_{d}\right)$ and the distortion parameters $\lambda$ and $\left(d_{x}, d_{y}\right)^{T}$. In the following section, we are going to show how this more complex formulation can be conveniently handled with a lifting trick.

\section{2 $\quad$ Lifting to $4 \mathrm{D}$ Space}

Lifting is a process in polynomial algebra which embeds a problem with nonlinear polynomial terms in a higher dimensional linear space. In our case, radially distorted points in the projective 2-plane $\mathbb{P}^{2}$ will be mapped to points in projective 3 -space $\mathbb{P}^{3}$. A distorted point with homogeneous coordinates $p_{d}=$ $\left(x_{d}, y_{d}, z_{d}\right)^{T} \in \mathbb{P}^{2}$ will be mapped to the point $\left(x_{d} z_{d}, y_{d} z_{d}, z_{d}^{2}, x_{d}^{2}+y_{d}^{2}\right)^{T} \in \mathbb{P}^{3}$. Hence, the projective 2-plane $\mathbb{P}^{2}$ is mapped to a quadric surface in $\mathbb{P}^{3}$ defined through $\left\{(x, y, z, w) \in \mathbb{P}^{3} \mid z w-x^{2}-y^{2}=0\right\}^{1}$. Interestingly and most importantly, the lifted distorted points can be mapped to the undistorted points by a fixed linear transformation, as we will derive shortly. Note that the same lifting scheme has been proposed by Barreto and Daniilidis [1] (see Eq. 7 in their

${ }^{1}$ Points of the form $\left(x_{d} z_{d}, y_{d} z_{d}, z_{d}, x_{d}^{2}+y_{d}^{2}\right)^{T}$ fulfill this equation $z w-x^{2}-y^{2}=0$ as can easily be verified by setting $x=x_{d} z_{d}, y=y_{d} z_{d}, z=z_{d}^{2}, w=x_{d}^{2}+y_{d}^{2}$. 
paper). Their derivation is however closely linked to the fundamental matrix, but we would like to highlight that this lifting trick can be applied independently of the type of multiple view constraint, i.e. it applies to homographies, trifocal tensors, etc as well. Furthermore, Barreto and Daniilidis assumed a known center of radial distortion. In the following, we show in detail how the same lifting scheme can be generalized to the case of unknown distortion center, resulting in a different linear transformation matrix than the one derived in [1], though.

Let us now present this lifting trick in detail, starting from the distortion model in Eq. 3. Simple algebraic manipulation of Eq. 3 leads to

$$
\left(\begin{array}{l}
x_{d} \\
y_{d}
\end{array}\right)+\lambda r^{2}\left(\begin{array}{l}
d_{x} \\
d_{y}
\end{array}\right)=\left(1+\lambda r^{2}\right)\left(\begin{array}{l}
x_{u} \\
y_{u}
\end{array}\right)
$$

which shows that the undistorted point $\left(x_{u}, y_{u}\right)^{T}$ is a scalar multiple of $\left(x_{d}, y_{d}\right)^{T}+$ $\lambda r^{2}\left(d_{x}, d_{y}\right)^{T}$. The scalar factor can be absorbed with a homogeneous representation

$$
p_{u}=\left(\begin{array}{c}
x_{u} \\
y_{u} \\
1
\end{array}\right) \cong\left(\begin{array}{c}
x_{d}+\lambda r^{2} d_{x} \\
y_{d}+\lambda r^{2} d_{y} \\
1+\lambda r^{2}
\end{array}\right)=\left[\begin{array}{rr}
1 & d_{x} \\
1 & d_{y} \\
& 1
\end{array}\right]\left(\begin{array}{l}
x_{d}-d_{x} \\
y_{d}-d_{y} \\
1+\lambda r^{2}
\end{array}\right)
$$

For additional generality and in order to stay closer to [1], let us represent the distorted point $p_{d}=\left(x_{d}, y_{d}, z_{d}\right) \in \mathbb{P}^{2}$ as an element of projective 2 -space. The previous equation Eq. 5 then becomes

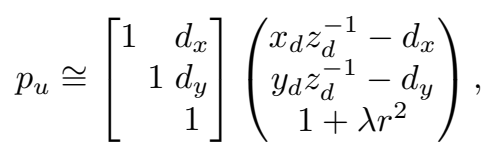

with $r^{2}=\left(x_{d} z_{d}^{-1}-d_{x}\right)^{2}+\left(y_{d} z_{d}^{-1}-d_{y}\right)^{2}$. Some further algebraic manipulations allow us to expose all the components due to the distorted point on the right hand side

$$
\begin{aligned}
& p_{u} \cong\left[\begin{array}{rr}
1 & d_{x} \\
1 & d_{y} \\
& 1
\end{array}\right]\left(\begin{array}{c}
x_{d} z_{d}^{-1}-d_{x} \\
y_{d} z_{d}^{-1}-d_{y} \\
1+\lambda\left(\left(x_{d} z_{d}^{-1}-d_{x}\right)^{2}+\left(y_{d} z_{d}^{-1}-d_{y}\right)^{2}\right)
\end{array}\right)
\end{aligned}
$$

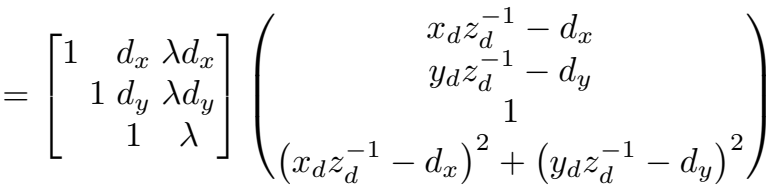

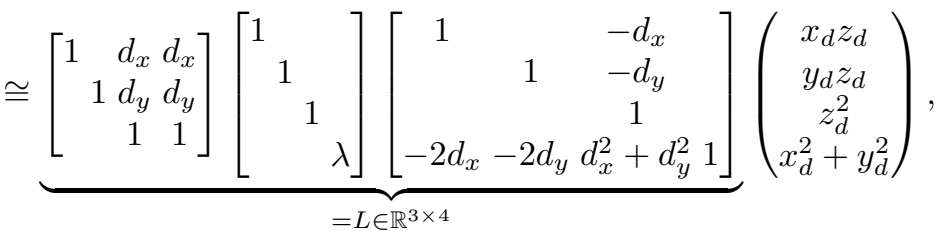


where in Eq. 8 the lifting trick has been used and Eq. 9 is equal to Eq. 8 up to a scale factor of $z_{d}^{2}$ which does not matter since $p_{u} \in \mathbb{P}^{2}$ is an element of projective 2 -space ${ }^{2}$. This derivation provides an important insight and leads to one of the main contributions of this paper. Eq. 9 shows that the undistorted homogeneous coordinates $p_{u}$ can be expressed by a $3 \times 4$ linear transformation $L$ applied from the left to the lifted data vector $\left(x_{d} z_{d}, y_{d} z_{d}, z_{d}^{2}, x_{d}^{2}+y_{d}^{2}\right)^{T} \in \mathbb{P}^{4}$. This linear algebraic representation has far reaching consequences. All multiple-view geometry entities, such as homographies or fundamental matrices, act on homogeneous coordinates of undistorted points. Unfortunately, if the input images are radially distorted, these entities are no longer applicable. However, these entities can be lifted to a higher dimensional space by multiplying them (either from the left and/or the right) with the 3-by-4 matrix $L$ thereby acting on radially distorted lifted coordinates. The matrix $L$ is a function of the radial distortion parameters and therefore also unknown. However, given sufficiently many distorted image observations, the lifted multiple view entities can be estimated nonetheless. This will be demonstrated in the following sections with the fundamental matrix.

\section{Single-Sided Radial Fundamental Matrix}

The fundamental matrix captures the projective relation between two camera views [10]. Given a homogeneous point correspondence $p_{u}$ and $q_{u}$ between two images of the same 3D point, the fundamental matrix relates these points by the constraint $q_{u}^{T} F p_{u}=0$. The fundamental matrix actually maps a point in one image to an epipolar line in the other image. Since neither $q_{u}$ nor $p_{u}$ can be the zero vector, $F$ has a non-trivial left and right nullspace. These nullspaces actually correspond to the two epipoles. The next section shows how the fundamental matrix can be extended to handle a radially distorted point measurement $p_{d}$ instead of an undistorted measurement $p_{u}$.

\subsection{Derivation of the Single-Sided Radial Fundamental Matrix}

Let us now assume that one of the two images is radially distorted, say the one where feature point $p_{u}$ has been observed. This means that only the radially distorted point $\left(x_{d}, y_{d}\right)^{T}$ is known. Thanks to the derivation in Sec. 3.2, we know how to handle this situation. A simple right-multiplication by $L$ lifts the fundamental matrix (on one side) to a $4 \mathrm{D}$ projective space which allows to use the radially distorted measurements

$$
0=q_{u}^{T} F p_{u}=q_{u}^{T} F L\left(\begin{array}{c}
x_{d} z_{d} \\
y_{d} z_{d} \\
z_{d}^{2} \\
x_{d}^{2}+y_{d}^{2}
\end{array}\right)=q_{u}^{T} \hat{F}\left(\begin{array}{c}
x_{d} z_{d} \\
y_{d} z_{d} \\
z_{d}^{2} \\
x_{d}^{2}+y_{d}^{2}
\end{array}\right),
$$

\footnotetext{
${ }^{2}$ Of course in practice, the measurement will be normalized such that $z_{d}=1$ and the formulas simplify slightly. Nevertheless, the more general representation is easier to interpret in terms of mappings between projective spaces.
} 
where the single-sided radial fundamental matrix $\hat{F}=F L \in \mathbb{R}^{3 \times 4}$ has been introduced. The decomposition $L=[I \mid 0]+\lambda\left(d_{x}, d_{y}, 1\right)^{T}\left(-2 d_{x},-2 d_{y}, d_{x}^{2}+d_{y}^{2}, 1\right)$ leads to another interesting representation of the single-sided radial fundamental matrix

$$
\hat{F}=F L=\left([F \mid 0]+F \lambda\left(\begin{array}{c}
d_{x} \\
d_{y} \\
1
\end{array}\right)\left(\begin{array}{lll}
-2 d_{x}-2 d_{y} d_{x}^{2}+d_{y}^{2} & 1
\end{array}\right)\right)
$$

\subsection{Properties of the Single-Sided Radial Fundamental Matrix}

Since the single-sided radial fundamental matrix $\hat{F}=F L$ is given as the product between the ordinary rank-2 fundamental matrix $F$ and the matrix $L$, its rank equals 2 . As a $3 \times 4$ matrix of rank 2 , the single-sided radial fundamental matrix has $3 \cdot 2+2 \cdot 4-2 \cdot 2-1=9$ degrees of freedom (minus one due to the scale ambiguity $)^{3}$.

Unfortunately, the number of parameters we are looking for equals 7 for the standard fundamental matrix plus 3 for the radial distortion parameters. Hence, there are 10 parameters but only 9 degrees of freedom in the single-sided radial fundamental matrix. This implies that there is a one-parametric family of perfectly valid solutions. Hence, given a single-sided radial fundamental matrix, it is not possible to uniquely extract the underlying fundamental matrix and the 3 radial distortion parameters. This is in contrast to previous work $[1,12]$ which assumed a known radial distortion center which decreased the number of parameters by 2. This allowed the unique extraction of all the 8 parameters. Nevertheless, in the remainder of this section, we will show that the epipoles are unique and how they can be extracted from the single-sided radial fundamental matrix even if the radial distortion center is unknown.

The extraction of the left epipole $e^{\prime}$ from the rank-2 matrix $\hat{F}$ is easy: Since $\hat{F}=F L$, both $\hat{F}$ and $F$ share the same left-nullspace and hence $e^{\prime}$ equals the left nullspace of $\hat{F}$. This nullspace can be easily computed e.g. with the singular-value decomposition of $\hat{F}$. The right epipole is more tricky since there is a two-dimensional right nullspace $N=\left[n_{1}, n_{2}\right] \in \mathbb{R}^{4 \times 2}$ of $\hat{F} \in \mathbb{R}^{3 \times 4}$, i.e. $\hat{F} N=0 \in \mathbb{R}^{3 \times 2}$. This nullspace can again be computed with the singularvalue decomposition of $\hat{F}$. The lifted coordinates of the distorted right epipole $e \in \mathbb{P}^{3}$ must lie in this nullspace since the undistorted epipole lies in the right nullspace of the standard fundamental matrix $F$ which is a factor of $\hat{F}=F L$. Hence, due to this fact and since the distorted coordinates are only defined up to scale, the lifted coordinates of the distorted epipole $e(\alpha)=\alpha n_{1}+(1-\alpha) n_{2}$ can be parametrized with one parameter $\alpha \in \mathbb{R}$. As described at the beginning of Sec. 3.2, valid points $(x, y, z, w) \in \mathbb{P}^{3}$ in the lifted space are restricted to a quadric surface defined through the equation $z w-x^{2}-y^{2}=0$. Plugging the

\footnotetext{
${ }^{3}$ A matrix $\mathbf{A} \in \mathbb{R}^{m \times n}$ of rank $r$ can be factorized $\mathbf{A}=\mathbf{B C}$ with $\mathbf{B} \in \mathbb{R}^{m \times r}$ and $\mathbf{C} \in \mathbb{R}^{r \times n}$. The matrix factors are unique up to a multiplication with a regular matrix $\mathbf{Q} \in \mathbb{R}^{r \times r}$, i.e. $\mathbf{A}=\mathbf{B} \mathbf{Q Q}^{-1} \mathbf{C}$ and as such $\mathbf{A}$ has $m r+r n-r^{2}$ degrees of freedom.
} 
one-parametric representation $e(\alpha)$ into this quadric equation yields a quadratic equation in $\alpha$ which can be solved easily in closed form. This results in two equally valid solutions for the distorted coordinates of the right epipole. Note that this is an inherent characteristics of the Fitzgibbon distortion model which always provides two possible distorted points, given the undistorted point and the radial distortion parameters ${ }^{4}$.

\subsection{Further Examples - Two-Sided Radial Fundamental and Homographies}

As already previously mentioned, the same lifting trick can be applied to other entities in multiple view geometry in the presence of radial distortion with unknown center of radial distortion. For example, the two-sided radial fundamental matrix where both images are radially distorted is given by left- and rightmultiplying the standard fundamental matrix with the transformations mapping lifted points to undistorted points, i.e.

$$
\left(x_{d}^{\prime}, y_{d}^{\prime}, 1, x_{d}^{\prime 2}+y_{d}^{\prime 2}\right) L^{\prime T} F L\left(x_{d}, y_{d}, 1, x_{d}^{2}+y_{d}^{2}\right)^{T}=0
$$

If both images have the same radial distortion, then $L=L^{\prime}$. This results in a $4 \times 4$ two-sided radial fundamental matrix which is again of rank 2 and has therefore $4 \cdot 2+2 \cdot 4-2 \cdot 2-1=11$ degrees of freedom. There are $7+3+3=13$ parameters ( 7 due to the standard fundamental matrix and twice times 3 parameters for the two distortion models), and again, there is no unique solution for the parameters. However, the two epipoles can be extracted analogously to the single-sided radial fundamental matrix.

Another example is given by a one-sided radial homography. Again multiplying the lifted coordinates $\tilde{x}$ of the distorted image from the left by $L$ yields $x^{\prime} \cong H L \tilde{x}$ and hence the one-sided radial homography $H L$ is a full rank $3 \times 4$ matrix. Both the two-sided radial fundamental matrix and the one-sided radial homography can be estimated with linear methods analogously to the algorithms presented next for the single-sided radial fundamental matrix.

\section{Single-Sided Radial Fundamental Matrix Estimation}

The algebraic epipolar constraint

$$
q^{T} \hat{F}\left(\begin{array}{llll}
x_{d} & y_{d} & 1 & x_{d}^{2}+y_{d}^{2}
\end{array}\right)^{T}=0
$$

can be rewritten using kronecker products [7] as

$$
\underbrace{\left(\begin{array}{llll}
x_{d} & y_{d} & 1 & x_{d}^{2}+y_{d}^{2}
\end{array}\right) \otimes q^{T}}_{A} \underbrace{\operatorname{vec}(\hat{F})}_{f}=0
$$

\footnotetext{
${ }^{4}$ Solving Eq. 3 for the distorted coordinates $\left(x_{d}, y_{d}\right)^{T}$ given $\left(x_{u}, y_{u}\right)^{T}, \lambda$, and $\left(d_{x}, d_{y}\right)^{T}$ asks for intersecting two conics which in this specific instance can have up to two solutions.
} 
From each correspondence, we obtain a different row vector $A_{i}$. Stacking 11 of these equations on top of each other we obtain an $11 \times 12$ matrix and $f$ must lie in the null space of that matrix, like in the 8-point algorithm for estimating the fundamental matrix. Similarly, rank two of the resulting matrix can be enforced via a singular value decomposition afterwards.

The ten point algorithm In an analogous way to the 7-point-algorithm for classical fundamental matrix estimation, we use one correspondence less than is required for the linear solution above and obtain a two-dimensional null-space spanned by $f_{1}$ and $f_{2}$. The true $f$ must thus be a linear combination of both, where we can fix one of the coefficients, since $f$ is only defined up to scale.

$$
f=\alpha f_{1}+f_{2}
$$

We now perform the inverse operation to vectorization and reassemble the matrix $\hat{F}$ from the vector $f$, and for convenience of notation, explicitely write down the columns:

$$
\hat{F}=\left(\hat{F}_{1} \hat{F}_{2} \hat{F}_{3} \hat{F}_{4}\right)
$$

We now choose alpha such that

$$
\operatorname{det}\left(\hat{F}_{1} \hat{F}_{2} \hat{F}_{3}\right)=0
$$

which is the same step as in the standard seven-point algorithm. Thus, from ten correspondences and one cubic determinant constraint we estimate the matrix $\hat{F}$. However, in the presence of noise, it is however not guaranteed that $\hat{F}$ will have rank two, since the last column of $\hat{F}$ can vary freely. Again, rank two of the resulting matrix can be enforced via SVD afterwards.

The nine point algorithm As mentioned above, in the ten-point-algorithm only the first three columns of $\mathrm{F}$ are forced to be in a $2 \mathrm{D}$ subspace, however, the last column could still vary freely in the presence of noise. Consequently, we might enforce also the last three columns of $F$ to be linearly dependent. To start, we can use only nine correspondences and obtain a 3D nullspace

$$
f=\alpha f_{1}+\beta f_{2}+f_{3}
$$

We now choose $\alpha$ and $\beta$ such that

$$
\operatorname{det}\left(\hat{F}_{1} \hat{F}_{2} \hat{F}_{3}\right)=0 \wedge \operatorname{det}\left(\hat{F}_{2} \hat{F}_{3} \hat{F}_{4}\right)=0
$$

These are two cubic equations in $\alpha$ and $\beta$ and according to Bezout's theorem there cannot be more than nine discrete solutions. The derivation of the exact solution is out of the scope of this paper, however the interested reader is refered to Groebner basis methods [11]. As argued before, there are nine degrees of freedom in $\hat{F}$ and so there can be no solution based on less than nine points. 


\section{Experiments}

In this section we demonstrate the usefulness of the presented formulation and prove empirically that the new model can cope with arbitrary distortion centers while earlier methods cannot. We first analyse this using synthetic data and then with real images. In the experiments, we use image pairs in which one image has known instrinsics and the other image has unknown focal length, radial distortion center and radial distortion coefficients. In the experiments with synthetic data we use random camera configurations, different distortion centers and different distortion parameters. Due to the lack of earlier methods for our setting, the results are compared to those obtained with the state of the art radial distortion solver from Kukelova et al.[12], although this latter method assumes the distortion center to be at the center of the image and also estimates distortion for both cameras. In contrast, in our setting, one of the images in each image pair has known intrinsics and the distortion center is not at the center of the image. We then test the algorithms with real world images which were taken with cameras that exhibit a significant level of distortion. We generated cropped versions of these images, so that the center of distortion would not lie at the center of the image.

\subsection{Evaluation with synthetic data}

The first set of tests for the semicalibrated case was performed using synthetic data. All tests with synthetic data were performed with a set of 100 random 3D points and 1000 generated random camera poses. The first camera was placed at the origin, with fixed parameters, pointed towards the set of 3D points. The 1000 random poses were generated for the second camera, by generating random translations, random rotations and random focal lengths, varying between $1 / 2$ and $2 \mathrm{x}$ the focal length of the first camera. For each camera pose we projected the $3 \mathrm{D}$ points on both cameras, distorted the points on the image of the second camera according to the distortion model in Eq. 3, setting the displacement of the distortion center to vary between 0 and the width/height of the image and using different values for the distortion coefficient. For each setting we computed the number of inliers with each algorithm. Results are presented in Fig. 1. We can see that, as the center of distortion is placed further away from the image center, the number of identified correspondences is constant for both implementations of our method, whereas method [12] increasingly fails to correctly identify the correspondences, as it does not correctly model the position of the distortion center.

\subsection{Test with real images}

To test the theory on real images we first matched a set of uncalibrated, distorted, cropped images to an image with known calibration parameters using different datasets. The undistorted images were cropped in such a way that the center of distortion would be located away from the center of the resulting image. 


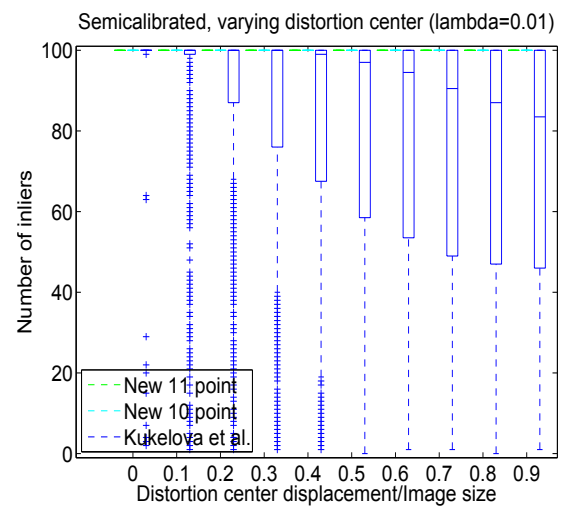

(a) Distortion parameter of $\lambda=0.1$.
Semicalibrated, varying distortion center (lambda $=0.1)$

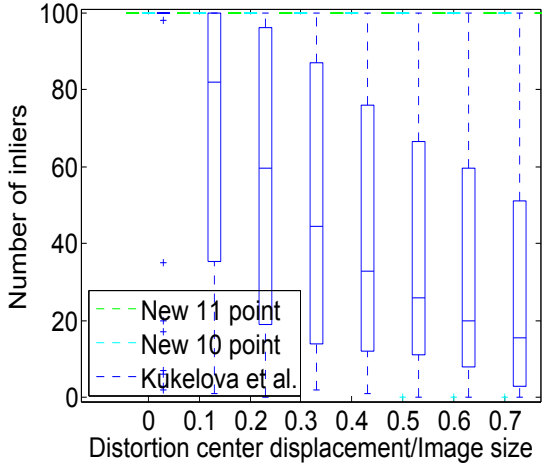

(b) Distortion parameter of $\lambda=0.01$.

Fig. 1: Boxplots of the number of inliers for 1000 randomly generated camera poses with varying distortion center. Note that our 11- and 10-point algorithms nearly always find all the 100 inliers.

To extract features in the images we used SURF[2], and then we computed a number of putative matches in each image pair by standard feature space matching. This produced a number of matches for each image pair, not all of which were correct correspondences. We then ran both the 11 point and 10 point implementations of our algorithm and [12], in a RANSAC framework with same parameters and constructing hypotheses on the same sample sets. In the end we computed the number of inliers with a threshold of 3 pixels. To obtain the epipolar error (used for classifying outliers) we computed the distance in pixels between a point and the epipolar line in the undistorted image. Before applying the point correspondences to the different algorithms, we normalise the image measurements similarly to the 8-point algorithm [8]. For the calibrated image we use the inverse of the camera intrinsics for the normalization, and for the uncalibrated/distorted/cropped one we use an initial estimate of the focal length, $f_{\text {guess }}=\frac{W / 2}{\tan \left(f o v_{\text {guess }} / 2\right)}$, where $W$ is the image width and $f o v_{\text {guess }}=50^{\circ}$ is an apriori estimate of the field of view. Furthermore, the image points are normalised with respect to the center of the image. As the normalisation is only performed to enhance the conditioning of the system, any similarity transformation is a valid normalisation in our formulation.

Results for one of the tested datasets are shown in Fig. 2 where we can see the inliers identified by method of [12] and our new method in an image pair where the image on the left has been previously undistorted with an offline camera calibration phase and the image on the right has unknown distortion parameters and was also cropped so that the distortion center is now in the upper part of the image. One can visualy see that our method is able to identify a higher number of inliers, especially in areas away from the distortion center. Fig. 3a shows a direct comparison of which inliers are identified by our new method and [12]. Again we 


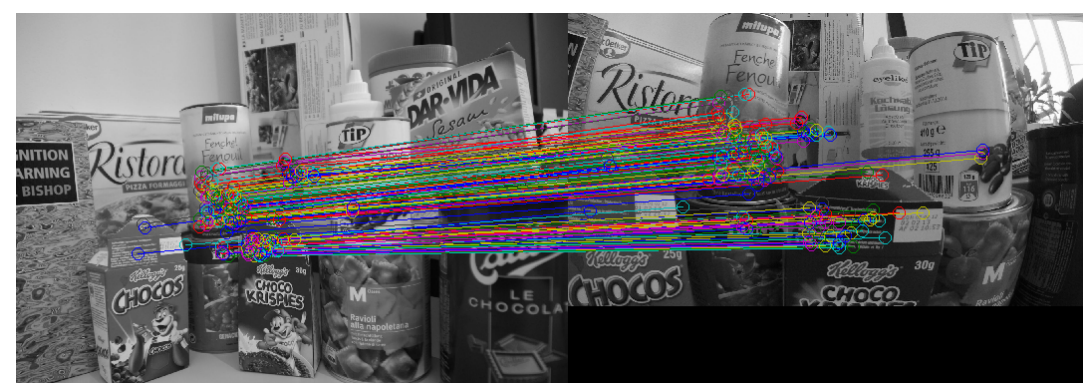

(a) 146 inliers identified by the method from Kukelova et al. [12]

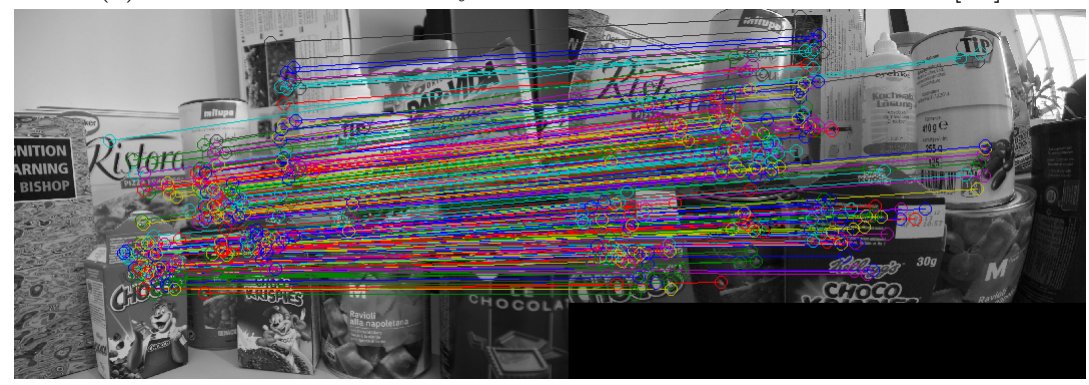

(b) 294 inliers identified by our new method.

Fig. 2: Results for dataset 'Shopping'.

can see that both methods identify inliers close to the center of distortion but our method identifies extra inliers away from the distortion center. Similar results can be obtained for different datasets in Fig. 3b and Fig. 3c. For the image pair in Fig. 3b the distorted image was cropped so that the distortion center was placed in the bottom right region of the image. For the image pair in Fig. 3c the distorted image was cropped so that the distortion center was placed in the top left region of the image. Also for these image pairs, the method from [12] found only a spatially confined set of correspondences near the center of distortion, whereas our method would be able to use more correspondences also far away from the center of distortion, where radial distortion is more severe. The inliers found by the method of [12] must be explained as an algebraic fit to the data, because the algorithm was not geometrically designed to cope with an unknown distortion center. To the best of our knowledge, the approach presented in this paper is the only one designed to handle epipolar geometry problems with fully unknown radial distortion.

\section{Conclusion}

We have shown that the lifting of image points into 4-space can consider the distortion center in a linear way. This allows for instance to generalize the radial fundamental matrix to the case of unknown distortion centers, facilitating now 


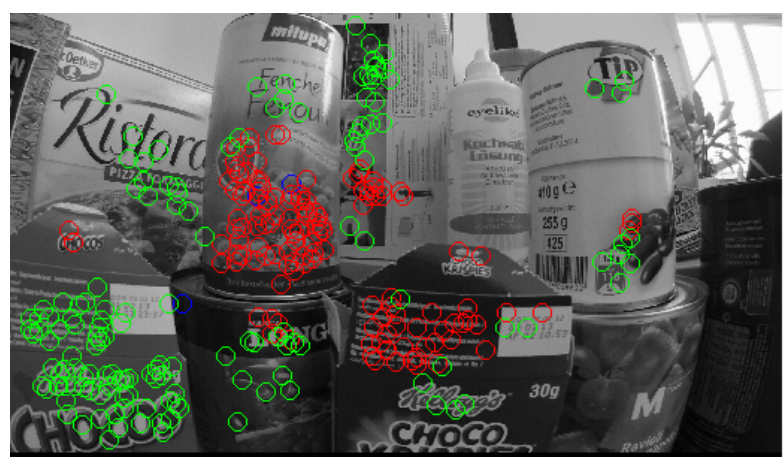

(a) Dataset 'Shopping'

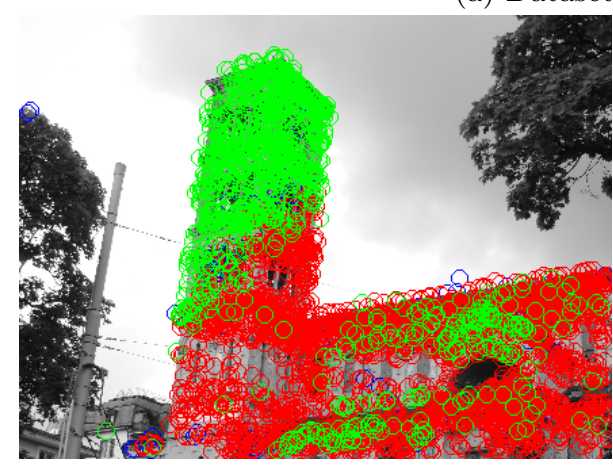

(b) dataset 'Church'

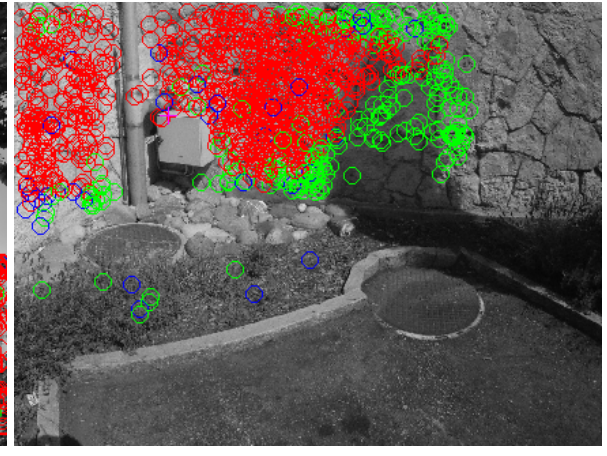

(c) dataset 'Corner'

Fig. 3: Comparison between our method and [12]: red are the inliers found by both methods; green are the extra inliers found by our method; blue are inliers found by the method from Kukelova et al. not found by our method.

practical use of the radial fundamental matrix even with cropped or zoomed images or more generally with images where the center of distortion is unknown. We have proven this by devising different algorithms to estimate the matrix from point correspondences and have shown results on real images that we believe cannot be obtained with any other existing framework. Furthermore, since a change of distortion center can be expressed linearly in 4-space, now the radial distortion model with unknown center can be applied to all multilinear multiple view relations, such as the trifocal tensor and homographies. Besides this, the insight about the distortion center might pave the way for a series of new minimal solvers with unknown distortion center. On top of this we believe that the new radial fundamental matrix can open the door to a theory of radial distortion self calibration, i.e. on top of focal length and principal point one could now look for the distortion coefficient and the distortion center when given multiple image pairs or image sequences, enforce some constraints (e.g. constant distortion center throughout a sequence) and so forth. 


\section{References}

1. Barreto, João P. and Kostas Daniilidis: Fundamental Matrix for Cameras with Radial Distortion International Conference on Computer Vision (ICCV) (2005) 625632

2. Bay, Herbert and Ess, Andreas and Tuytelaars, Tinne and Van Gool, Luc: SURF: Speeded Up Robust Features Computer Vision and Image Understanding (CVIU) 3 (110) (2008) 346-359

3. Brown, D. C.: Close-range camera calibration Photogrammetric Engineering 8 (37) (1971) 855-866

4. Claus, D. and Fitzgibbon, A. W.: A Rational Function Lens Distortion Model for General Cameras Computer Vision and Pattern Recognition (CVPR) (2005) 213219

5. Faugeras, O. and Luong, Q. and Maybank, S.: Camera self-calibration: Theory and experiments European Conference on Computer Vision (ECCV) 588 321-334

6. Fitzgibbon, A.W.: Simultaneous linear estimation of multiple view geometry and lens distortion Computer Vision and Pattern Recognition (CVPR) 1 (2001) 125132

7. Andrea Fusiello: A matter of notation: Several uses of the Kronecker product in 3D computer vision Pattern Recognition Letters 15 (28) (2007) 2127-2132

8. Hartley, R.: In defense of the eight-point algorithm Pattern Analysis and Machine Intelligence (PAMI) 6 (19) (1997) 580-593

9. Hartley, R. and Kang, Sing Bing: Parameter-Free Radial Distortion Correction with Center of Distortion Estimation Pattern Analysis and Machine Intelligence (PAMI) 8 (29) (2007) 1309-1321

10. Hartley, R. and Zisserman, A.: Multiple View Geometry in Computer Vision Cambridge University Press (2004)

11. Kukelova, Zuzana and Bujnak, Martin and Pajdla, Tomas: Automatic Generator of Minimal Problem Solvers European Conference on Computer Vision (ECCV) (2008) 302-315

12. Kukelova, Zuzana and Byröd, Martin and Josephson, Klas and Pajdla, Tomas and Aström, Kalle: Fast and robust numerical solutions to minimal problems for cameras with radial distortion Computer Vision and Image Understanding (CVIU) 2 (114) (2010) $234-244$

13. Hongdong $\mathrm{Li}$ and Richard Hartley: A Non-iterative Method for Correcting Lens Distortion from Nine-Point Correspondences In Proc. OmniVision05, ICCVworkshop (2005)

14. Longuet: A computer algorithm for reconstructing a scene from two projections Nature 293 (1981) 133-135

15. Quan-Tuan Luong and Olivier D. Faugeras: The fundamental matrix: Theory, algorithms, and stability analysis International Journal of Computer Vision (IJCV) 1 (17) (1996) 43-75

16. Micusik, B. and Pajdla, T.: Estimation of omnidirectional camera model from epipolar geometry Computer Vision and Pattern Recognition (CVPR) 1 (2003) 485490

17. Thirthala, S. and Pollefeys, M.: Multi-view geometry of $1 \mathrm{D}$ radial cameras and its application to omnidirectional camera calibration International Conference on Computer Vision (ICCV) 2 (2005) 1539-1546 